\title{
Device for rheometry, impedance spectroscopy, and electrochemistry on fluid electrodes
}

Cite as: Rev. Sci. Instrum. 90, 025112 (2019); https://doi.org/10.1063/1.5031126

Submitted: 28 March 2018 . Accepted: 02 December 2018 . Published Online: 25 February 2019

Aditya Narayanan (D), Frieder Mugele (D), and Michael H. G. Duits
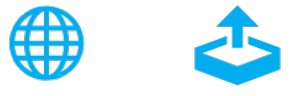

View Online

Export Citation

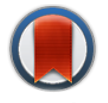

\section{ARTICLES YOU MAY BE INTERESTED IN}

A compact and robust cooling laser system for an optical strontium lattice clock

Review of Scientific Instruments 90, 023109 (2019); https://doi.org/10.1063/1.5063552

Erratum: "Image-plate sensitivity to x rays at 2 to $60 \mathrm{keV}$ " [Rev. Sci. Instrum. 90, 013506 (2019)]

Review of Scientific Instruments 90, 029902 (2019); https://doi.org/10.1063/1.5092602

\section{A multi-mode digital holographic microscope}

Review of Scientific Instruments 90, 023705 (2019); https://doi.org/10.1063/1.5066556

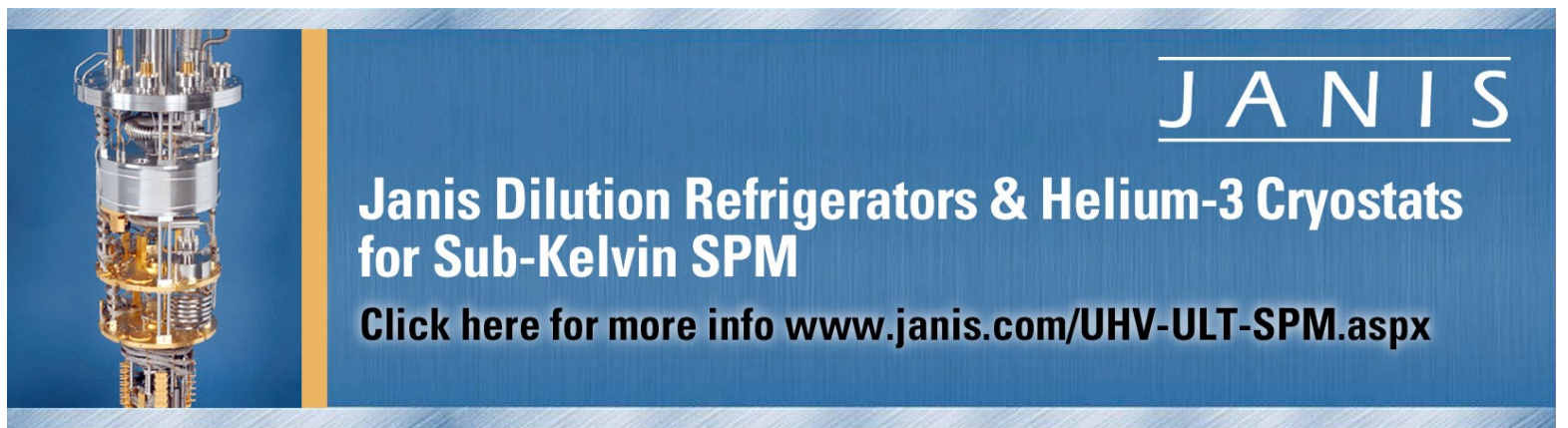




\title{
Device for rheometry, impedance spectroscopy, and electrochemistry on fluid electrodes
}

\author{
Cite as: Rev. Sci. Instrum. 90, 025112 (2019); doi: 10.1063/1.5031126 \\ Submitted: 28 March 2018 - Accepted: 2 December 2018 • \\ Published Online: 25 February 2019
}

Aditya Narayanan, (D) Frieder Mugele, (D) and Michael H. G. Duits ${ }^{\text {a) }}$

\section{AFFILIATIONS}

Physics of Complex Fluids Group, MESA+ Institute, University of Twente, P.O. Box 217, 7500 AE Enschede, The Netherlands

\begin{abstract}
${ }^{a}$ Author to whom correspondence should be sent: m.h.g.duits@utwente.nl
ABSTRACT

We describe the extension of a rheometer to enable in situ impedance spectroscopy and electrochemical cycling. Key advantages of this instrument over traditional flow-channel based methods for studying fluid electrodes are the possibilities to monitor the rheological properties during cycling as well as to control the mechanical history of the sample. We describe two electrochemical configurations of the instrument, allowing fluid electrodes to be studied as full and half-cells. To demonstrate the systems' capabilities, we present characterizations of 4 different fluid electrode systems.
\end{abstract}

Published under license by AIP Publishing. https://doi.org/10.1063/1.5031 126

\section{INTRODUCTION}

Novel solutions for grid energy storage and production such as Semi Solid Flow Batteries (SSFBs), ${ }^{1}$ flow supercapacitors, $^{2}$ and flow capacitive mixing generators ${ }^{3}$ are receiving a strong interest in current research and development. ${ }^{4}$ Semi-solid flow architectures use fluid electrodes with active particles that store charge either through faradic or (pseudo) capacitive processes and conductive particles that impart electronic conductivity dispersed in an electrolyte solution. The conductive particles are typically colloidal carbon blacks. They transfer electrons through a percolated (agglomerate) network, which can even be formed during flow. In the quiescent state, these networks also prevent active particle sedimentation by providing a finite yield stress to the suspension. 5,6

One major challenge that comes with the development of these systems lies in the dispersed state of the colloidal particles. In contrast to traditional batteries, a fluid electrode can adapt its structure to externally imposed fields such as gravity, mechanical shear, or electrochemical potential. The response of the system can be a modification of the fluid structure without altering the particles themselves, but it can also take place via changes in the particles' surfaces and colloidal interactions. These changes at the particle and the overall structure level can strongly influence the performance of the fluid electrode. Not only shear rate but also shear history has been found to significantly change the electronic conductivity and rheological properties of the electrodes due to the restructuring of particle agglomerates. ${ }^{5,7,8}$ Electrochemical operation of SSFB fluids was shown to induce surface layers such as Solid Electrolyte Interphase (SEI) on the particles, thereby strongly affecting the low frequency electrical impedance of the fluid. ${ }^{6,9}$ In flow capacitive mixing electrodes, electrochemically induced changes in salt concentration ${ }^{4}$ can affect colloidal interactions and hence the agglomeration behavior ${ }^{10}$ and microstructure.

Each of these examples underlines that the understanding (and subsequent performance optimization) of colloidal fluid electrodes requires the ability to control both mechanical and electric fields, as well as the availability of in situ characterization tools. At the minimum, two different types of electrochemical sensing and control are needed: the ability to apply a DC bias for electrochemical cycling and a small AC perturbation for impedance spectroscopy, both on suitable counter electrodes. Besides that, the rheological capabilities should include at least measurements of the yield stress and the viscosity flow curve. Such versatility cannot be offered by existing instruments; flow-channel setups capable of electrochemical testing ${ }^{1,2,9,11,12}$ do not allow a well-defined control over mechanical history, while existing combined rheology-impedance spectroscopy instruments ${ }^{5,8,13-16}$ do not allow electrochemical cycling. 
In this paper, we describe a novel setup capable of simultaneous rheometry, impedance spectroscopy, and electrochemical cycling. For electrochemical cycling, two (counter) electrode configurations are described. The first, applicable to semi solid flow battery electrodes, uses a lithium foil as a counter electrode. The second uses a larger volume of a second fluid as the counter electrode. This configuration is particularly suited for studying Flow Super Capacitor (FSC) electrodes that require a very high surface area counterelectrode and other flow systems in a full cell configuration.

\section{GLOBAL SETUP}

Figure 1 shows a schematic of the apparatus. It consists of a parallel plate geometry for rheometry with both shearing surfaces (CC1 and $\mathrm{CC} 2$ ) acting also as current collectors for impedance spectroscopy. This geometry was chosen to achieve a uniform electric field across the sample (FE1), at the expense of a constant strain field. The rheometer applies torques and measures strains on the upper geometry.

The bottom stationary shearing surface $(\mathrm{CC} 2)$ is a perforated disk that allows the fluid electrode to interact with a counter electrode below, which is used for electrochemical cycling. A polymeric membrane that is permeable to solvent and ions but not to the colloidal particles separates the two electrodes. Depending on the application, two counter electrode configurations are possible. The first is the semisolid flow battery half-cell which uses a lithium metal foil as the counter electrode. The second configuration is the flow supercapacitor/semi solid flow battery full cell which uses a second fluid as a counter electrode.

In either case, the fluid electrode under study (between $\mathrm{CC} 1$ and $\mathrm{CC} 2$ ) is in direct contact with two ionically blocking but electronically reversible metal current collectors. This configuration is particularly advantageous for impedance measurements as it allows a direct probing of the upper fluid electrode's electronic resistance (besides some interfacial contributions), i.e., the low frequency limit of the real impedance. ${ }^{5,6}$

\section{A. Detailed description}

A Haake Rheostress RS 600 (geometrically equivalent to the RS 6000 model) controlled stress rheometer (not shown) forms the base and frame of the instrument. The upper rheometry geometry is a combination of a rotating body belonging to the rheometer (a "rotor") and a disk. The former is a commercial (Haake) stainless steel electro-rheology rotor with a ceramic inter-connect to provide electrical isolation from the rheometer spindle. To the bottom of the rotor, a $60 \mathrm{~mm}$ stainless steel disk (CC1) was glued using VBlocks for alignment. A very small amount of (non-conductive) cyanoacrylate superglue was used to achieve a strong bonding between the rotor and disk, while preserving electrical contact. To electrically connect this upper rheometry geometry to the outside, with minimal electrical noise and mechanical resistance, a steel solvent trap is used with mercury as a working fluid. The variation in impedance of the mercury connection due to rotation was measured to be less than $10 \mathrm{~m} \Omega$. The contribution to the measured torque was negligible for all measurements to be shown. The stationary part of the solvent trap is placed on a splittable 3D printed solvent trap base that sits on a polypropylene housing.

All other parts (see Fig. 1) are assembled into a polypropylene housing which is placed on the base of the rheometer. A thin stainless-steel disk with $(\sim 1 \mathrm{~mm}$ radius) perforations (CC2) is used as the bottom shearing surface. Above it is a metal contact ring which is electrically connected to the outside via a screw. A permeable polymeric membrane is placed below the perforated disk to electronically separate the (upper) fluid electrode FE1 from the lower components (including the counter electrode).

The placement of the lower components depends on the configuration. For the semi-solid flow battery half-cell configuration, a lithium foil disk is placed below the membrane on top of a third current collector plate (CC3). A thick lithium disk is used to give the membrane mechanical support. For the flow supercapacitor/semi solid flow battery full cell configuration, a stainless-steel mesh is placed below the

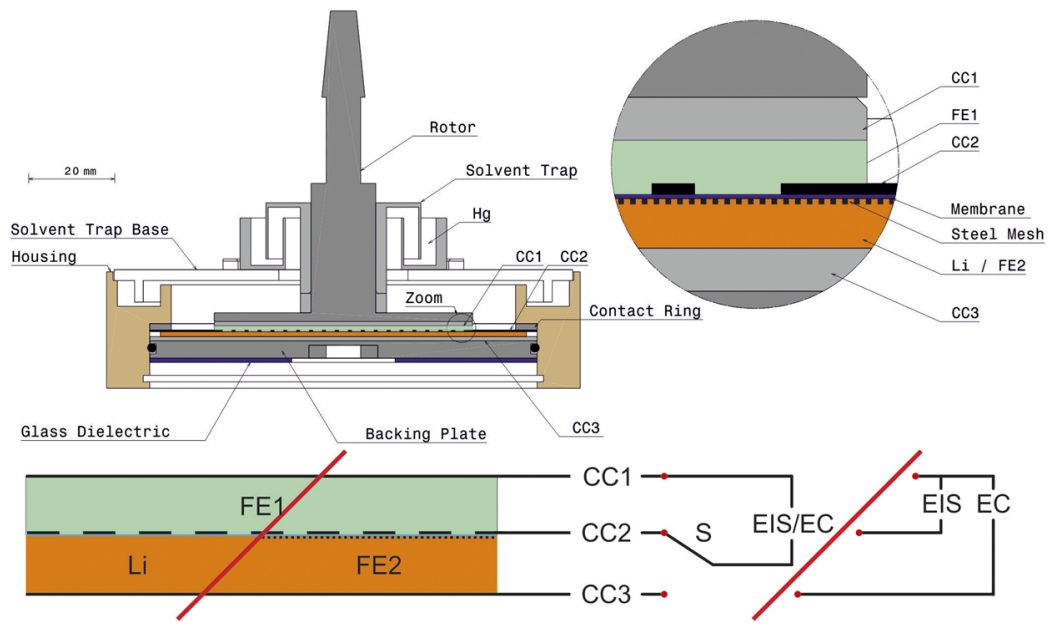

FIG. 1. Top: Cross section of the apparatus. The encircled inset shows a zoomed-in view. Bottom: Schematic of the measurement geometry and external electric circuit in two configurations, separated by a slash: the LHS corresponds to a half-cell, and the RHS corresponds to a full cell. Meaning of symbols-CC1: upper current collector and shearing surface (grey), CC2: current collector and lower shearing surface (black), CC3: lower current collector (light grey), EC: electrochemical cycling, EIS: electrical impedance spectroscopy, FE1: fluid electrode 1 (green), FE2: fluid electrode 2 (orange), Li: lithium foil (orange), and S: switch. 


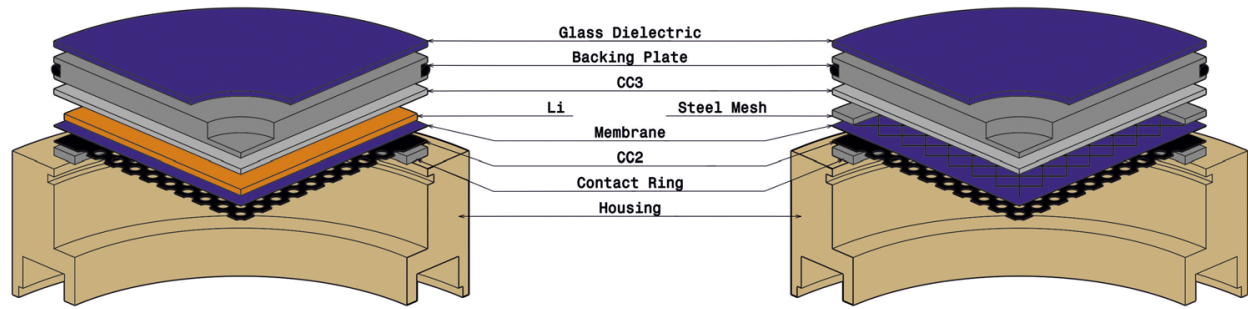

FIG. 2. Sections demonstrating the assembly of the device in (left) half-cell and (right) full-cell configurations. The polypropylene housing is held upsidedown in this stage.

membrane to provide mechanical support. A spacer (not shown) and the third current collector along with the mesh create a compartment that is filled with the second fluid electrode (FE2).

A metal backing plate with an O-ring seals the cell and is electrically connected to the outside via a screw. The backing plate is also used to align the lower rheometry geometry with the rheometer base and the spindle, using an alignment pin. Below the backing plate is a thin glass disk that acts as a dielectric, isolating the counter electrode from the rheometer body. The relatively high thermal conductivity of the glass (compared to other dielectrics) allows for effective temperature measurement and control from the rheometer base. A retainer spring (not shown) in a groove of the polypropylene housing presses it and all the lower geometry parts onto the rheometer base.

There are two possible configurations for electrochemistry and impedance spectroscopy that can be used with the instrument: single and dual potentiostat. The single potentiostat configuration uses relays to switch between electrochemical measurement and control. During electrochemical cycling, switch S is "down" and the potential of CC1 (and the fluid electrode) is controlled versus CC3, while CC2 is left floating. During spectroscopic measurements, switch S is "up" and the impedance spectra are measured between $\mathrm{CC} 1$ and $\mathrm{CC} 2$ with $\mathrm{CC} 3$ floating. The second dual potentiostat configuration allows simultaneous impedance measurement and electrochemical control. One potentiostat controls the potential of $\mathrm{CC} 2$ versus $\mathrm{CC} 1$ for impedance spectroscopy and measures the currents through it. The second does the same for CC3 (versus CC1). It is important that the potentiostat in control of $\mathrm{CC} 3$ has a lower bandwidth than that of the $\mathrm{AC}$ impedance measurement to prevent cross feedback. Thus, the impedance measurement is typically limited to higher frequencies unless the potentiostat connected to $\mathrm{CC} 3$ is set to open circuit.

\section{B. Assembly and sample loading}

The lower rheometry geometry is first assembled, in a top down manner, into the inverted polypropylene base, as shown in Fig. 2. After the contact ring, perforated plate (CC2) and solvent wetted membrane are placed, and the counter electrode is assembled. In the case of the SSFB half-cell, the lithium foil disk and then the third current collector are placed. For the SSFB/FSC full-cell, the steel mesh with spacer is placed. A side screw (not shown) is used to hold the pressed spacer and mesh against the membrane, flattening it.
An excess of the fluid electrode 2 (not shown) is introduced, and the third current collector is pressed onto the spacer. Excess slurry is then wiped off. For both configurations, the backing plate is then pressed against the third current collector. The glass dielectric and the retaining spring (not shown) are placed, and the bottom geometry is installed on the rheometer base. The rotor is then screwed on the rheometer spindle.

After zeroing the rheometer gap, the sample (fluid electrode 1) is introduced. The gap is closed, excess sample is trimmed off, and the solvent trap is assembled and filled with mercury.

\section{Rheological verification}

The modified measurement geometry was verified using two viscosity standards at various settings for the gap, i.e., the distance between $\mathrm{CC} 1$ and $\mathrm{CC} 2$. For all gaps, the calibration oils gave viscosities that remained constant within $5 \%$ (standard deviation) as the shear rate was varied (logarithmically) from 10 to $1000 \mathrm{~s}^{-1}$. Figure 3 shows the (shear-rate averaged) viscosity as a function of the gap height; the variation is less than $10 \%$. These results are similar to those obtained with the standard bottom plate of the instrument.

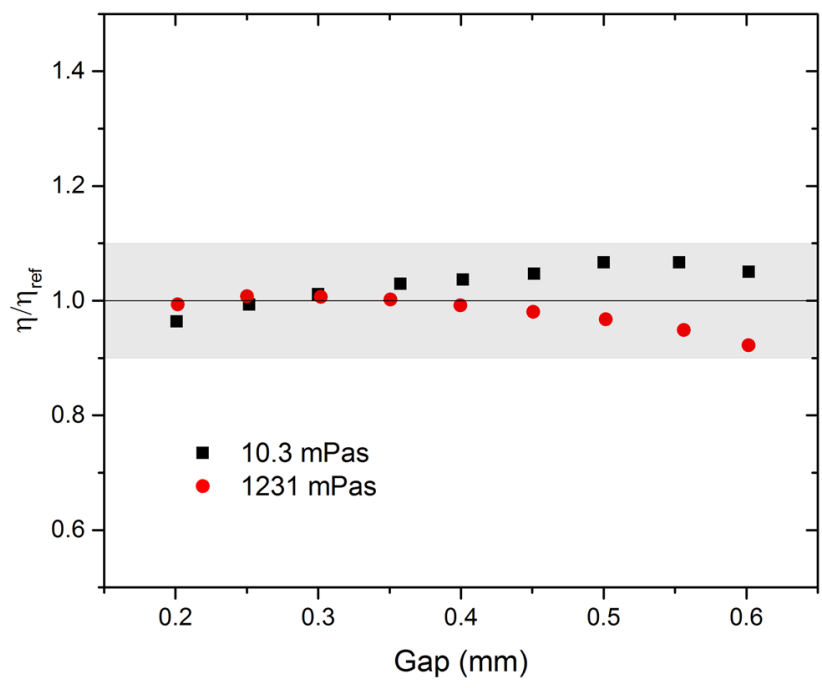

FIG. 3. Normalized viscosity versus plate-plate gap for two calibration oils. The black line is the reference viscosity. The gray shaded area represents a $10 \%$ error 


\section{APPLICATION TO FLOW SYSTEMS}

We demonstrate the versatility of the instrument by presenting the evolution of the fluid yield stress and Low Frequency Real Impedance (LFRI) on electrochemical cycling for four fluid electrode systems. The reason for choosing LFRI values is that, unlike fitted values, they do not rely on models of the electrical impedance spectroscopy (EIS) spectra (shown in the supplementary material) which can vary with the system and charge state. We have previously demonstrated ${ }^{6}$ that the LFRI is a reasonable measure of the trends in the electronic resistance.

\section{A. SSFB fluid electrodes in half-cell configuration}

Figure 4 shows the evolution of the yield stress and Low Frequency Real Impedance (LFRI) of a SSFB anolyte [lithium titanate; Lithium Titanate Oxide (LTO) based] and catholyte (lithium nickel cobalt manganese oxide; LiNCM based) before and during electrochemical cycling. The suspensions were composed of $5 \mathrm{wt}$ \% active material and $1 \mathrm{wt}$. \% conductive additive (Ketjen Black), suspended in an electrolyte solution. The details of the electrode materials and preparation are given in the supplementary material.

To demonstrate the importance of mechanical pre-shear (and subsequent rest), we first consider the samples in the electrochemically pristine state. We applied a pre-shear at the indicated rate for $200 \mathrm{~s}$ and allowed a rest time of $200 \mathrm{~s}$. For both anolyte [Fig. 4(a)] and catholyte [Fig. 4(b)], it is observed that a higher pre-shear rate leads to a higher yield stress and a lower LFRI. Rates higher than $1000 \mathrm{~s}^{-1}$ do not lead to a significant enhancement of the effect. This indicates a complete breakup of the carbon black network, before allowing the primary units to agglomerate again during the subsequent rest, ${ }^{5,13}$ thereby defining a reproducible reference state. Thus a $200 \mathrm{~s} 1000 \mathrm{~s}^{-1}$ pre-shear (followed by a $200 \mathrm{~s}$ rest) was applied before all measurements.

In subsequent electrochemical cycling experiments, the single potentiostat configuration was used. Each fluid electrode (FE1) was cycled in the half cell configuration versus a lithium foil in separate experiments. Cycling was performed at a constant current until the indicated potential was reached. Experimental details hereof are included in the supplementary material. For the anolyte, the low frequency real impedance shows very strong alternations with the state of charge. Additionally, there is an increase in LFRI per (charged or discharged) state, when electrochemically cycled. The yield stress also shows strong alternations in the opposite direction, with a lower yield stress correlating with a higher impedance and vice versa. Cycling the catholyte also has significant effects on the yield stress with it lowered in the charged state. The effects on the catholyte are however much weaker than those of the anolyte. The increases in the impedance and decreases in the yield stress (observed within the accepted operating range of the materials ${ }^{4,9}$ ) are very detrimental to SSFB fluid electrodes. The observed trends can be partially explained by formation of surface layers (solid electrolyte interface) on the conductive carbons during charging that partially dissolve on discharging. ${ }^{6}$ It is of note that the effect of different pre-shears on the pristine electrode yield stress and LFRI is similar in magnitude to that of a full charge discharge cycle. This highlights the importance of a well-defined mechanical history to fluid electrode measurements.

\section{B. FSC fluid electrode in full-cell configuration}

Figure 5 shows the evolution of the yield stress and LFRI of a non-aqueous (propylene carbonate based) and an aqueous flow super capacitor fluid electrode when electrochemically cycled. The fluid electrodes contained conductive carbon (Ketjen Black; 2 wt.\% for the aqueous and $1.5 \mathrm{wt} \%$ for the non-aqueous) suspended in electrolyte solutions. They contained no electrochemically active particles. The details of the electrode materials and preparation are given in the supplementary material. The counter electrode in both cases was of a much larger volume ( 4 times of the same fluid electrode). The fluid electrodes under study were pre-sheared at $1000 \mathrm{~s}^{-1}$ for $200 \mathrm{~s}$ followed by a rest time of $200 \mathrm{~s}$ before all measurements.

During electrochemical cycling experiments, the dual potentiostat configuration was used. The fluid electrode under study was held at the indicated potential versus the lower fluid electrode for $20 \mathrm{~min}$ to cycle it. The non-aqueous fluid electrode's LFRI shows strong alternations, with the negative potential state consistently presenting higher LFRI values. The alternations diminish with the cycle number in the $2.7 \mathrm{~V}$ window and increase in the $3.5 \mathrm{~V}$ one. In all cases, however, the LFRI is actually lower than that of the pristine state. The yield stress does not show any correlation with the
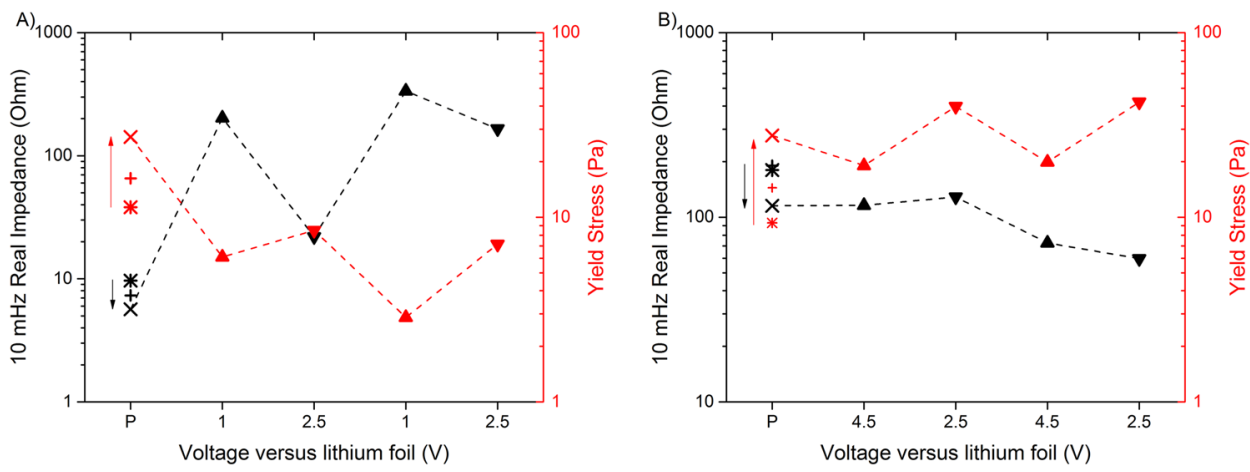

FIG. 4. Low frequency real impedance and yield stress of anolyte (a) and catholyte (b) versus the charge state where $P$ indicates the pristine state, and the other states are defined via the reached voltage. The up triangles represent the charged state $(1 \mathrm{~V}$ for anolyte and $4.5 \mathrm{~V}$ for catholyte), and the down triangles represent the discharged state. The arrows mark increasing pre-shear rates $\left(100,316,1000 \mathrm{~s}^{-1}\right)$ for the pristine state. See the supplementary material (Fig. S2) for impedance spectra. 

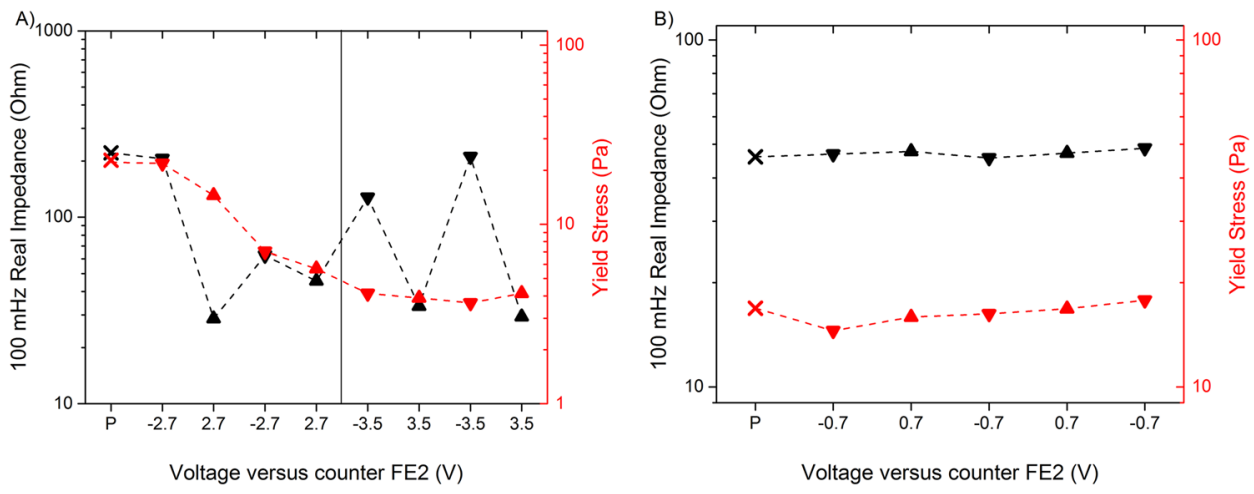

FIG. 5. Evolution of the LFRI and yield stress of a non-aqueous (a) and aqueous (b) flow super capacitor fluid electrode when cycled. the down triangles represent negative voltage states, and the up triangles represent positive voltage states. In the non-aqueous case, the vertical line demarcates two voltage regimes. See the supplementary material (Figs. S3 and S4) for impedance spectra.
LFRI. Instead it decreases every half cycle up to some lower limit. The aqueous fluid electrode on the other hand does not exhibit any significant effect of electrochemical cycling. As these systems do not show detrimental effects (and even show somewhat positive ones), they may be viable candidates for FSC electrodes.

\section{SUMMARY}

We have described a modification to a (typical) commercial rheometer that extends its measurement capabilities for colloidal suspensions to in situ impedance spectroscopy and electrochemical cycling. Example experiments first confirm the importance of controlled pre-shear in order to define a reproducible reference state and second show strong effects of electrochemical cycling on the fluid electrodes' electrical and mechanical properties. This highlights the usefulness and relevance of the setup to study suspension fluid electrodes. As the apparatus can be set up with a modest amount of effort on commercial rheometers, we expect it to be of use to others involved in fluid electrode research. We have provided downloadable CAD files of all the parts online.

\section{SUPPLEMENTARY MATERIAL}

See supplementary material for the detailed descriptions of the materials, sample preparation, and experimental protocols.

\section{ACKNOWLEDGMENTS}

The research leading to these results has received funding from the European Union Seventh Framework
Programme (No. FP7/2007-2013) under Grant Agreement No. 608621.

\section{REFERENCES}

${ }^{1}$ M. Duduta, B. Ho, V. C. Wood, P. Limthongkul, V. E. Brunini, W. C. Carter, and Y. M. Chiang, Adv. Energy Mater. 1, 511 (2011).

${ }^{2}$ V. Presser, C. R. Dennison, J. Campos, K. W. Knehr, E. C. Kumbur, and Y. Gogotsi, Adv. Energy Mater. 2, 911 (2012).

${ }^{3}$ S. Porada, D. Weingarth, H. Hamelers, M. Bryjak, V. Presser, and P. Biesheuvel, J. Mater. Chem. A 2, 9313 (2014).

${ }^{4}$ K. B. Hatzell, M. Boota, and Y. Gogotsi, Chem. Soc. Rev. 44, 8664 (2015).

${ }^{5}$ A. Narayanan, F. Mugele, and M. H. G. Duits, Langmuir 33, 1629 (2017).

${ }^{6}$ A. Narayanan, D. Wijnperlé, F. Mugele, D. Buchholz, C. Vaalma, X. Dou, S. Passerini, and M. Duits, Electrochim. Acta 251, 388 (2017).

${ }^{7}$ G. Ovarlez, L. Tocquer, F. Bertrand, and P. Coussot, Soft Matter 9, 5540 (2013).

${ }^{8}$ M. Youssry, L. Madec, P. Soudan, M. Cerbelaud, D. Guyomard, and B. Lestriez, Phys. Chem. Chem. Phys. 15, 14476 (2013).

${ }^{9}$ E. Ventosa, G. Zampardi, C. Flox, F. La Mantia, W. Schuhmann, and J. Morante, Chem. Commun. 51, 14973 (2015).

${ }^{10}$ Y. Zhang, A. Narayanan, F. Mugele, M. A. C. Stuart, and M. H. Duits, Colloids Surf., A 489, 461 (2016).

${ }^{11}$ Z. Li, K. C. Smith, Y. Dong, N. Baram, F. Y. Fan, J. Xie, P. Limthongkul, W. C. Carter, and Y.-M. Chiang, Phys. Chem. Chem. Phys. 15, 15833 (2013).

${ }^{12}$ S.-i. Jeon, H.-r. Park, J.-g. Yeo, S. Yang, C. H. Cho, M. H. Han, and D. K. Kim, Energy Environ. Sci. 6, 1471 (2013).

${ }^{13}$ A. Helal, T. Divoux, and G. H. McKinley, Phys. Rev. Appl. 6, 064004 (2016). ${ }^{14} \mathrm{M}$. Youssry, L. Madec, P. Soudan, M. Cerbelaud, D. Guyomard, and B. Lestriez, J. Power Sources 274, 424 (2015).

${ }^{15}$ J. J. Richards, J. B. Hipp, J. K. Riley, N. J. Wagner, and P. D. Butler, Langmuir 33, 12260 (2017).

${ }^{16}$ J. J. Richards, N. J. Wagner, and P. D. Butler, Rev. Sci. Instrum. 88, 105115 (2017). 